\title{
OTIOLIOMTS
}

Revista de economía, empresa y sociedad

Dosier sobre economía colaborativa (y II)

Nuevas estrategias y dimensiones alternativas de la economía de plataforma

Coordinador: Lluís Alfons Garay Tamajón

NUEVAS FORMAS DE TRABAJO

\section{H futuro es colectivo y los colectivos son el futuro}

\section{Albert Gañigueral Bagó}

Ouishare

RESUMEN La evolución hacia la empresa-red y la fragmentación del trabajo que se deriva incrementa el número de personas trabajadoras autónomas con relaciones intermitentes entre varios empleadores y con sus posibles compañeros de trabajo. En este contexto emergen los «colectivos de autónomos" o "colectivos de independientes» en contraposición a la narrativa de que los trabajadores autónomos están aislados entre sí. Estos colectivos organizan luchas contra los algoritmos, luchas por los derechos laborales, mutualizan recursos materiales y digitales e incluso se presentan al mercado bajo una marca unificada sin ser una empresa. Al ser formas emergentes de organización, se enfrentan a retos acerca de su reconocimiento como actores en el diálogo social e incluso se les acusa de actuar como cárteles bajo el prisma de la libre competencia. Si bien sus formas actuales es probable que no sean las formas definitivas, podemos intuir que los "colectivos de autónomos» tienen y tendrán un papel relevante para definir las formas de trabajar y de vivir en el futuro.

PALABRAS CLAVE plataformas digitales; trabajadores de plataforma; economía gig; colectivos; autónomos 


\title{
The future is collective and the collectives are the future
}

\begin{abstract}
The evolution towards the network company and the resulting fragmentation of work increases the number of self-employed persons with intermittent relationships between various employers and with their potential co-workers. In this context, "collectives of the self-employed" emerge in contrast to the narrative that self-employed workers are isolated from each other. These collectives organise fights against algorithms, fights for labour rights, pool material and digital resources and even present themselves to the market under a unified brand without being a company. As emerging forms of organisation, they face challenges regarding their recognition as actors in social dialogue and are even accused of acting as cartels under the prism of free competition. Although their current forms are probably not the definitive forms, we can intuit that the "collectives of the self-employed" have and will have a relevant role in defining the ways of working and living in the future.
\end{abstract}

KEYWORDS digital platforms; platform workers; gig economy; collectives; self-employed

\section{Introducción}

Desde las últimas décadas del siglo XX, la «empresa-red» (Artiles, 1994) se ha convertido en el modelo paradigmático de organización productiva empresarial, fomentando la externalización de todo aquello que no se considera el centro del negocio (core business). Esto ha derivado en una mayor temporalidad en el empleo. Millones de personas ya no configuran sus vidas laborales alrededor de un empleo tradicional de 9 a 5 en un único empleador durante un período prolongado de tiempo (ver imagen 1). La irrupción de las plataformas digitales laborales (Cañigueral, 2020), que reducen los costes de transacción y coordinación para encontrar el talento que las organizaciones necesitan (Moore, 2014), ha acelerado aún más las tendencias de externalización preexistentes. Con ello la vida y la carrera laboral de un número creciente de personas trabajadoras se ha fragmentado (Bersin, 2019) y las fronteras del tiempo y el espacio de trabajo se han difuminado. En este artículo se explora cómo estas personas trabajadoras, con vidas laborales fragmentadas, se unen para cubrir sus necesidades laborales y vitales a la vez que defienden sus derechos laborales en un entorno narrativo y normativo que no les favorece.

\section{Imagen 1. El trabajo ya no es lo que era}

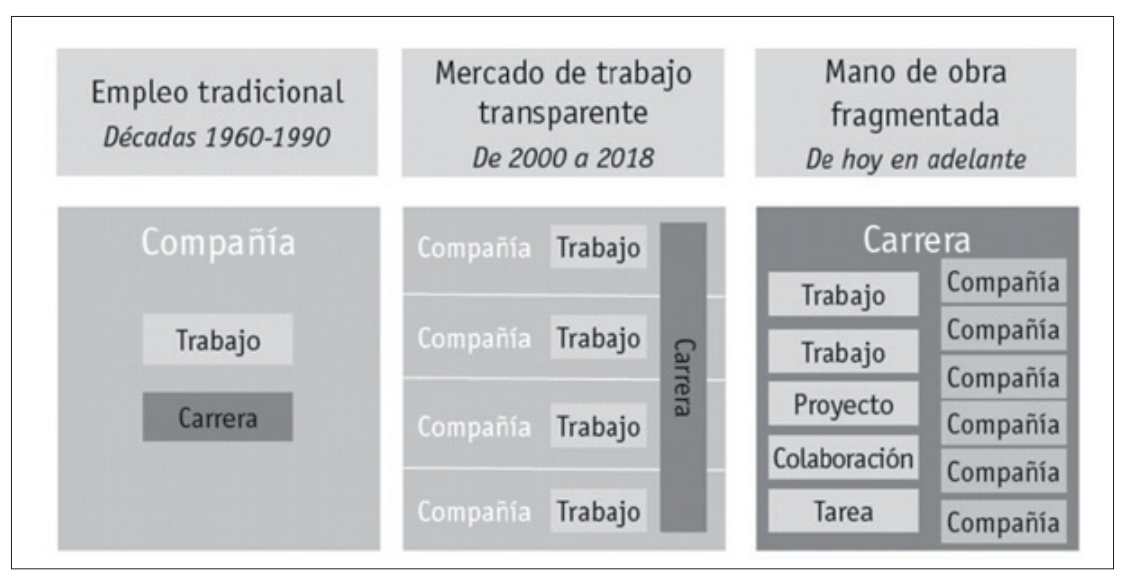

Fuente: adaptación de Josh Bersin 


\section{Trabajadores fragmentados del mundo, juníos!}

«iProletarios de todos los países, uníos!» es un lema político incluido en el Manifiesto del Partido Comunista, escrito por Karl Marx y Friedrich Engels, y una de las consignas más famosas y representativas de la causa del comunismo a nivel internacional. Si lo adaptamos al nuevo paradigma laboral, podríamos decir algo como: «iTrabajadores fragmentados del mundo, uníos!». Tras el estudio de las nuevas formas laborales durante unos años, una de las conclusiones imprevistas a las que he llegado es que el protagonista del futuro del trabajo no es el trabajador individual (fragmentado, autónomo o independiente), sino que son los «colectivos de autónomos» o "colectivos de independientes". La calidad de la experiencia laboral es muy diferente para el trabajador autónomo aislado, que es menos productivo y tiene problemas de salud más a menudo, que para los trabajadores autónomos agrupados. Así como las tecnologías digitales facilitan la fragmentación del trabajo, son estas mismas tecnologías las que facilitan la unión (Heimans y Timms, 2018) de los trabajadores autónomos en colectivos. Para aclarar lo comentado, se presentan en la tabla 1 varios ejemplos de cómo estas personas trabajadoras se unen mediante diversidad de formatos por diversos intereses.

\section{Tabla 1. Resumen de colectivos de trabajadores autónomos}

\begin{tabular}{|c|c|c|}
\hline Función principal & Descripción & Ejemplos \\
\hline Resistencia algorítmica & $\begin{array}{l}\text { Coordinarse para forzar un comportamiento concreto } \\
\text { de los algoritmos de la plataforma. }\end{array}$ & $\begin{array}{l}\text { Uber Surge Club, } \\
\text { Instagram Engagement Pods }\end{array}$ \\
\hline Sindical & $\begin{array}{l}\text { Defensa de los derechos laborales de las personas } \\
\text { trabajadoras en un sector de actividad concreto. } \\
\text { Operan a nivel local o nacional y se coordinan a nivel } \\
\text { supranacional. }\end{array}$ & $\begin{array}{l}\text { RidersXDerechos, Acua Chile, } \\
\text { Independants.co }\end{array}$ \\
\hline Mutualismo & $\begin{array}{l}\text { Mutualizar servicios compartidos (gestoría, seguros, } \\
\text { acceso a formación especializada, etc.). A menudo en } \\
\text { forma cooperativa. }\end{array}$ & Smart, Coopaname, CoopArt \\
\hline Espacio físico & $\begin{array}{l}\text { Espacios de trabajo compartido para una actividad } \\
\text { sectorial. Coworking de nicho. Comunidad. }\end{array}$ & $\begin{array}{l}\text { The Wing, The Writers Room, } \\
\text { The Qube, Nest City Lab }\end{array}$ \\
\hline Aprendizaje & $\begin{array}{l}\text { Compartir dudas y conocimiento en espacios mayo- } \\
\text { ritariamente digitales. Existen en diversos grados de } \\
\text { formalidad. }\end{array}$ & $\begin{array}{l}\text { SinOficina, StackOverflow, Meetups, } \\
\text { Grupos de Facebook, etc. }\end{array}$ \\
\hline Acceso al mercado & $\begin{array}{l}\text { Agrupación de trabajadores autónomos bajo una mis- } \\
\text { ma marca, sector de actividad, valores, etc. Aportan } \\
\text { continuidad al entorno laboral fragmentado. }\end{array}$ & $\begin{array}{l}\text { Ouishare, Happy-Dev, MyWaySpain, } \\
\text { Hoxby }\end{array}$ \\
\hline $\begin{array}{l}\text { Propiedad de los medios } \\
\text { de trabajo }\end{array}$ & $\begin{array}{l}\text { Plataformas cooperativas donde los trabajadores } \\
\text { son propietarios del código y de la gobernanza del } \\
\text { proyecto. }\end{array}$ & $\begin{array}{l}\text { CoopCycle, Mensakas, Up\&Go, } \\
\text { Stocksy, Eva }\end{array}$ \\
\hline
\end{tabular}




\subsection{Cuatro ámbitos de unión en mayor profundidad}

\section{Colectivos para la resistencia algorítmica}

Hecho el algoritmo, hecha la trampa. Mediante estrategias suficientemente elaboradas, mediante un trabajo colectivo, es posible hackear el algoritmo de la plataforma. Por ejemplo, en Instagram existen los grupos de compromiso (engagement pods) de influencers como una forma emergente de resistencia (O'Meara, 2019). Los grupos acuerdan comentar, compartir o interactuar entre sí, sin importar el contenido. De esta manera, el algoritmo de Instagram prioriza el contenido de los participantes y lo muestra a un público más amplio. Por otro lado, las estrategias de los conductores de Uber para disparar de manera artificial el surge pricing («multiplicador de precio») han sido bastante comentadas en los medios americanos. El incremento de precios se activa cuando hay una gran demanda de viajes, como cerca de un aeropuerto o después de un concierto o un partido de fútbol, y poca oferta. Los conductores pueden activar el surge pricing al apagar y encender sus aplicaciones de manera coordinada. Por ejemplo, se acercan a la zona del estadio y cinco minutos antes del final de un partido apagan las apps. En ese momento los espectadores empezaran a pedir viajes y al no existir oferta suficiente sube el precio. Cinco minutos más tarde vuelven a activar la aplicación y pueden ganar más dinero por el mismo trabajo que iban a realizar. En canales de YouTube de conductores para aplicaciones hablan del «Surge Club» (Hamilton, 2019), en referencia al Fight Club (El club de la lucha), y a la vez piden que Uber reflexione acerca de su política de remuneración.

\section{Colectivos de carácter sindical o neosindical}

Históricamente, uno de los elementos clave para la mejora de las condiciones de los trabajadores ha sido su organización colectiva y la acción sindical. En el caso de muchos de los trabajadores atípicos (ej.: Las Kellys de los hoteles) y de los trabajadores de plataforma, que no comparten ni espacio ni tiempo de trabajo, resulta algo más difícil, pero no imposible. Hoy en día la coordinación puede materializarse mediante la creación de un grupo de WhatsApp, mediante el que se puede proponer una huelga 2.0 (Shenker, 2019). En el ámbito de los trabajadores de plataforma, los primeros en organizarse fueron los trabajadores de las plataformas bajo demanda, contribuyendo al incremento de su visibilidad respecto a otros colectivos. En España, a mitades de 2017, los repartidores de Deliveroo convocaron la primera huelga de trabajadores de una plataforma digital. Los riders de Deliveroo crearon Ridersxderechos como plataforma para agrupar a los trabajadores que se sumaron a la huelga.

Fue también Ridersxderechos quien lideró el segundo encuentro mundial de asociaciones de riders en Barcelona en abril de 2019. Hubo participación de otras asociaciones como la argentina APP Sindical (Asociación de Personal de Plataformas), la inglesa IWW Couriers Network o la francesa CLAP. Todas ellas se unen bajo la Transnational Courier Federation para defender sus derechos local y globalmente. Colectivos similares han emergido allá donde las plataformas de trabajo bajo demanda han empezado a funcionar. Debido a la heterogeneidad de los trabajadores de plataforma (Cañigueral, 2020) (incluso dentro de una misma plataforma o sector) emergen diversidad de grupos que representan a mensajeros con objetivos y puntos de vista diversos. Además de Ridersxderechos y SindicatoRiders, colectivos que denuncian la relación de falsos autónomos con las plataformas y piden una relación de empleo por cuenta ajena, existen otras asociaciones, como AsoRiders, Asociación Profesional de Riders Autónomos (APRA) y AutoRiders, que se posicionan a favor de seguir siendo autónomos y centran su actividad en mejoras operativas de las plataformas y de protección de los trabajadores autónomos. El conflicto entre los diversos colectivos es visible en los medios de comunicación (Cózar, 2020).

Otro de los colectivos que más y mejor se han organizado han sido los conductores de Uber y similares, con agrupaciones de trabajadores como United Private Hire Drivers, Independent Drivers Guild, Drivers United, Gig Workers Rising o AcuaChile, por mencionar algunos de los más activos. A finales de enero de 2020, representantes de conductores de 23 países se reunieron en Londres para crear la International Alliance of App-based Transport Workers (IAATW) y así llevar su lucha colectiva a una escala global (Varghese, 2020), a la misma escala que muchas de estas plataformas. El mapa "Leeds Index of Platform Labour Protest» 
(Trappmann et al., 2020) recopila y analiza más de 300 protestas y conflictos de los trabajadores de plataformas bajo demanda a escala global. En la mayoría de las instancias, los cambios en el sistema de pagos han sido la causa principal.

Los medios y la literatura se centran mucho en los ejemplos en riders y conductores por ser los más mediáticos, pero el fenómeno de los neosindicatos abarca todos los sectores de actividad. Nunca fue ni tan fácil ni tan necesario organizarse entre los trabajadores. La National Domestic Workers Alliance es un gran grupo de trabajadoras del hogar en Estados Unidos. En España las camareras de hotel, conocidas como kellys, tienen grupos locales en las principales ciudades turísticas. También hay grupos para redactores en medios digitales (Digital Writers Union) o youtubers (FairTube). Otro tipo de trabajadores de plataforma que se ha organizado de manera más visible han sido los freelancers mediante colectivos como Freelancers Union (Estados Unidos) o Independants.co (Francia). Todos se han organizado colectivamente para mejorar sus condiciones de trabajo.

\section{Colectivos de carácter mutualista}

Los trabajadores independientes también se han unido para mutualizar riesgos y repartir gastos en grupos de intereses similares. En algunos casos acaban desarrollando unos servicios WorkerTech (Cañigueral, 2019) de carácter más cooperativo. En Reino Unido el caso de Breadfunds fue de los pioneros. Se trata de un grupo de 25 a 50 personas que contribuyen con algo de dinero cada mes a un fondo para poder apoyar a cualquiera de estos miembros cuando no pueden trabajar por una enfermedad o lesión. En Francia WeMind y OtherWise siguen la misma lógica. También en Francia la ley de las CAE (coopératives d'activités et d'emploi) ha permitido el nacimiento de proyectos como Port Parallèle, Coopaname u Oxalis; donde uno se hace socio y trabajador asalariado de la cooperativa. El «salario» y el volumen de cotizaciones dependerá de los proyectos facturados como trabajador independiente, pero es un trabajador asalariado a ojos de la administración, bancos, etc. En España existen CoopArt o Smart Iberia que, amparadas bajo la ley de cooperativas de Andalucía, actúan de manera similar a una CAE. Smart Iberia forma parte del grupo europeo Smart con sede central en Bélgica y con más de 120.000 socios en toda Europa. Las cooperativas se encargan de la administración y se llevan un $\%$ por todo ello.

\section{Colectivos para acceder al mercado}

Mi propia vida laboral es fragmentada haciendo de consultor, divulgador, formador, escritor, etc. a lo largo del año para varios clientes en Europa y América principalmente. En este "caos», lo que me aporta continuidad, sentido de pertenencia e identidad laboral es el colectivo Ouishare. Son mis compañeros/as de aventuras y de cuando en cuando también mis compañeros/as de trabajo en diversos proyectos.

Estar acompañado de esta manera permite aprender más rápido, mutualizar algunos temas comunes (espacio de coworking, gestoría, herramientas informáticas, etc.). Nos pasamos trabajos los unos a los otros y cuidamos colectivamente la marca y la reputación de manera que es más fácil para todos conseguir otros trabajos individuales o colectivos. La última evolución con la que se está experimentando son los «PODS» (Basterfield, 2018) y los «TetraPod» (Trepat, 2021). Existen otros grupos similares a Ouishare. Happy Dev (desarrolladores por toda Francia), Collectif Cosme (en Burdeos como agrupación de freelancers de la comunicación), Enspiral (Nueva Zelanda) o Colab.coop (Estados Unidos), por mencionar algunos. Estos colectivos no suelen ser muy grandes ni tienen ambición de crecer. Es gente que se une por afinidad en valores y por tener habilidades complementarias en un ámbito profesional concreto. Luego existen colectivos de mayor escala y menor foco en los valores, más cercanos a una red de expertos que a una comunidad. Destaco Hoxby, Moonlightwork, Contra, MyWaySpain o Communo. Estas agrupaciones ofrecen a las empresas su grupo de freelancers altamente seleccionados. Algo similar a una agencia o un bufete, pero sin empleados y con capacidad de acceder a talento muy diverso en todo el mundo. 


\subsection{Observaciones adicionales}

A la luz de los ejemplos, algunas observaciones adicionales:

- Este tipo de agrupaciones son un fenómeno relativamente reciente en el ámbito de los trabajadores de plataforma. Hay otros sectores, como el ámbito creativo y artístico, donde los trabajadores han vivido con relaciones laborales fragmentadas desde hace muchos años. Estudiar en mayor profundidad estos sectores puede ser relevante para anticipar la evolución y los retos que se van a presentar en los colectivos de trabajadores de plataforma.

- Los colectivos de carácter sindical y de acceso al mercado, al no encajar legalmente en un formato de sindicato tradicional al no ser asalariados, se enfrentan a varios retos. Por un lado, su nivel de reconocimiento como interlocutores en el diálogo social no es siempre el que sería deseable (Gyulavári, 2020). Por otro lado, con una interpretación estrecha de las leyes de competencia, se les está acusando de tener comportamientos de tipo cártel (ej.: fijación de precios mínimos) (DCCA, 2020). Recientemente la Comisión Europea ha emitido una iniciativa para permitir a los trabajadores autónomos negociar colectivamente sin ser obstaculizados por la ley de competencia. Este trabajo deberá ser seguido de cerca.

- Las plataformas digitales laborales (UpWork/Freelancer.com) están empezando a aceptar no solo individuos, sino también grupos de freelancers y agencias. Las empresas también deberán empezar a incluir la contratación de colectivos, no solo de personas, en su gestión de talento. Como dice Xavier Damman de Open Collective: «Cuando contratas a alguien de una comunidad, estás contratando a mucho más que al individuo en particular. También te beneficias de la inteligencia colectiva de la(s) comunidad(es) a la(s) que pertenece» (Damman, 2017).

\section{Conclusiones}

Como ha quedado demostrado con la revisión de iniciativas, la colectivización de las personas trabajadoras con vidas y carreras laborales fragmentadas es un fenómeno emergente, en crecimiento y en movimiento. No creo que la forma actual de relación entre empleadores, plataformas y trabajadores independientes sea la forma final en la que se configurará el futuro del trabajo. En algunos aspectos estos colectivos se podrían comparar con los colectivos comerciales de la Edad Media que eran los gremios: compañeros de profesión que se unen para aprender, organizarse y defenderse. Del mismo modo que han surgido los neosindicatos, en próximas iteraciones pueden emerger «neogremios digitales" y tener un rol importante en el futuro del trabajo de los trabajadores autónomos. El estratega John Hagel lo ha resumido con un «From the Gig Economy to the Guild Economy» (Hagel, 2020), que no tiene una traducción simple al castellano. Sin duda los "colectivos de autónomos» tienen y tendrán un papel relevante para definir las formas de trabajar y de vivir en el futuro. Será recomendable seguir de cerca su evolución.

\section{Bibliografía}

ARTILES, A. (1994). «La empresa red: un modelo de división del trabajo entre empresas». Papers, vol. 44, pág. 87. DOI: https://doi.org/10.5565/rev/papers.1747

BASTERFIELD, S. (2018). «P's in a pod: Purpose, Proximity \& Product - the journey of the Golden Pandas». Enspiral Tales [en línea] [fecha de consulta: 28 de enero de 2021]. Disponible en: https://medium.com/enspiraltales/ps-in-a-pod-purpose-proximity-product-the-journey-of-the-golden-pandas-ab3c77e29abf

BERSIN, J. (2019). «The Pixelated Workforce: A Job For Almost Everyone». Josh Bersin [en línea] [fecha de consulta: 28 de enero de 2021]. Disponible en: https://joshbersin.com/2019/06/the-pixelated-workforce-a-jobfor-almost-everyone/. 
CAÑIGUERAL, A. (2019). El mercado digital a debate: Plataformas, trabajadores, derechos y WorkerTech. Fundación COTEC. ISBN: 978-84-92933-42-6

CAÑIGUERAL, A. (2020). El trabajo ya no es lo que era: Nuevas formas de trabajar, otras maneras de vivir. Penguin Random House Grupo Editorial, España. ISBN: 9788417992057

DCCA (2020). «Commitment decision on the use of a minimum hourly fee». DCCA [en línea] [fecha de consulta: 28 de enero de 2021]. Disponible en: https://www.en.kfst.dk/nyheder/kfst/english/decisions/20200826-commitment-decision-on-the-use-of-a-minimum-hourly-fee-hilfr/

CÓZAR, C. (2020). "La guerra de 'riders': quién es quién en la batalla por los derechos de los repartidores de Glovo». Economía Digital [en línea] [fecha de consulta: 28 de enero de 2021]. Disponible en: https://www. economiadigital.es/finanzas/la-guerra-de-riders-quien-es-quien-en-la-batalla-por-los-derechos-de-los-repartidores-de-glovo_20113708_102.html

DAMMAN, X. (2017). «From firms to collectives». Open Collective [en línea] [fecha de consulta: 28 de enero de 2021]. Disponible en: https://medium.com/open-collective/from-firms-to-collectives-c139ae27a4ee

MOORE, G. (2014). «La naturaleza de la empresa (75 años después)». Reinventar la empresa en la era digital. Alianza Editorial, pág. 51-62.

GYULAVÁRI, T. (2020). "Collective rights of platform workers: The role of EU law». Maastricht journal of European and comparative law, vol. 27, núm. 4, pág. 406-424. DOI: https://doi.org/10.1177/1023263X20932070

HAGEL, J. (2020). «From the Gig Economy to the Guild Economy». John Hagel [en línea] [fecha de consulta: 28 de enero de 2021]. Disponible en: https://www.johnhagel.com/from-the-gig-economy-to-the-guild-economy/

HAMILTON, I. A. (2019). «Uber drivers are reportedly colluding to trigger «surge» prices because they say the company is not paying them enough». Business Insider [en línea] [fecha de consulta: 28 de enero de 2021]. Disponible en: https://www.businessinsider.com/uber-drivers-artificially-triggering-surge-prices-reports-abc7-2019-6

HEIMANS, J.; TIMMS, H. (2018). New Power: How Power Works in Our Hyperconnected World --and how to Make it Work for You. Doubleday. ISBN: 9780385541114

O'MEARA, V. (2019). «Weapons of the chic: Instagram influencer engagement pods as practices of resistance to Instagram platform labor». Social media + society, vol. 5, núm. 4, págs. 205630511987967. DOI: https://doi. org/10.1177/2056305119879671

SHENKER, J. (2019). "Strike 2.0: how gig economy workers are using tech to fight back». The Guardian [en línea] [fecha de consulta: 28 de enero de 2021]. Disponible en: http://www.theguardian.com/books/2019/aug/31/ the-new-resistance-how-gig-economy-workers-are-fighting-back

TRAPPMANN, V. et al. (2020). «Trends in Collective Action, Organising and Mobilisations in the Platform Economy». 32nd Annual Meeting [en línea]. SASE. Disponible en: https://sase.confex.com/sase/2020/meetingapp.cgi/Paper/15890

TREPAT, A. (2021). «High-trust teams to navigate the futureS of work». Medium, 2021 [en línea] [fecha de consulta: 28 de enero de 2021]. Disponible en: https://aliciatrepatpont.medium.com/high-trust-teams-to-survivethe-future-of-work-d807e3949ea6

VARGHESE, S. (2020). «Gig economy workers have a new weapon in the fight against Uber». WIRED UK [en línea] [fecha de consulta: 28 de enero de 2021]. Disponible en: https://www.wired.co.uk/article/gig-economy-uberunions

Citación recomendada: CAÑIGUERAL BAGÓ, Albert. El futuro es colectivo y los colectivos son el futuro. Oikonomics [en línea]. Mayo 2021, no. 15, pp. 1-8. ISSN: 2339-9546. DOI: https://doi. org/10.7238/o.n15.2101 


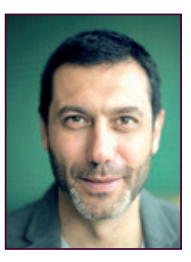

\section{Albert Cañigueral} albert@ouishare.net Ouishare

Albert Cañigueral es el Connector Ouishare para España y América Latina. En 2011 fundó el blog Consumo Colaborativo y, desde entonces, es considerado uno de los mayores referentes de la economía de plataforma en español. Ha publicado recientemente el libro El trabajo ya no es lo que era: nuevas formas de trabajar, otras maneras de vivir (Conecta, 2020). Trabaja como explorador, consultor y divulgador en el ámbito de la economía de plataforma y actualmente se ha centrado sobre todo en el futuro del trabajo, el impacto de las plataformas digitales en las ciudades y las innovaciones regulatorias.

Los textos publicados en esta revista están sujetos -si no se indica lo contrario- a una licencia de Reconocimiento 4.0 Internacional de Creative Commons. Puede copiarlos, distribuirlos, comunicarlos públicamente, hacer obras derivadas siempre que reconozca los créditos de las obras (autoría, nombre de la revista, institución editora) de la manera especificada por los autores o por la revista. La licencia completa se puede consultar en https://creativecommons.org/licenses/by/4.0/deed.es_ES.

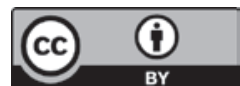

